\title{
Production of glycine-derived ammonia as a low-cost and long-distance
}

\section{antibiotic strategy by Streptomyces}

3

4

Mariana Avalos ${ }^{1}$, Paolina Garbeva ${ }^{2}$, Jos M. Raaijmakers ${ }^{1,2}$, Gilles P. van Wezel ${ }^{1,2 *}$.

5

${ }^{1}$ Institute of Biology, Leiden University, Sylviusweg 72, 2333 BE Leiden, The Netherlands.

7

${ }^{2}$ Netherland Institute of Ecology, Department of Microbial Ecology, Droevendaalsesteeg 10,

6708 PB Wageningen, The Netherlands.

9

*Author for correspondence. Tel: +31 71 5274310; Email: g.wezel@biology.leidenuniv.nl 
15

\section{ABSTRACT}

Soil-inhabiting streptomycetes are Nature's medicine makers, producing over half of all known antibiotics and many other bioactive natural products. However, these bacteria also produce many volatile compounds, and research into these molecules and their role in soil ecology is rapidly gaining momentum. Here we show that streptomycetes have the ability to kill bacteria over long distances via air-borne antibiosis. Our research shows that streptomycetes do so by producing surprisingly high amounts of the low-cost volatile antimicrobial ammonia, which travels over long distances and antagonises both Grampositive and Gram-negative bacteria. Glycine is required as precursor to produce ammonia, and inactivation of the glycine cleavage system annihilated air-borne antibiosis. As a resistance strategy, E. coli cells acquired mutations resulting in reduced expression of the porin master regulator OmpR and its cognate kinase EnvZ, which was just enough to allow them to survive. We further show that ammonia enhances the activity of the more costly canonical antibiotics, suggesting that streptomycetes adopt a low-cost strategy to sensitize competitors for antibiosis over longer distances. 


\section{INTRODUCTION}

Volatile Compounds (VC) are small molecules with high vapor pressure and low molecular weight that easily diffuse through air, water or soil(Schmidt et al 2015, Schulz and Dickschat 2007). VCs have a broad activity-spectrum, acting as infochemicals, growth-promoting or inhibiting agents, modulators of quorum sensing and drug resistance or as a carbon-release valve, influencing their neighbor's behavior and phenotypes such as stress response, colony morphology, biofilm, virulence and pigment production(Audrain et al 2015, Kai et al 2009, Kim et al 2013, Nijland and Burgess 2010, Que et al 2013). In soil, VCs play important roles in inter- and intra-species interactions(Schulz-Bohm et al 2017).

Actinobacteria are one of the largest bacterial phyla present in soil(Barka et al 2016, Cordovez et al 2015). They are known as Nature's medicine makers (Hopwood 2007b), with the ability to produce bioactive secondary metabolites that work as among others antibiotics, anticancer, antifungal, anthelmintic and immunosuppressant agents(Barka et al 2016, Bérdy 2012, Hopwood 2007a). Streptomycetes alone produce half of all known antibiotics used in the clinic. Streptomycetes are also prolific producers of volatile compounds, often with unknown functions(Citron et al 2015, Schöller et al 2002). In terms of antimicrobial bioactivity of VCs, information is primarily available on their activity as antifungals(Cordovez et al 2015, Wang et al 2013). A rare example of a volatile organic compounds (VOC) with antibacterial activity is the sesquiterpene albaflavenone produced by Streptomyces albidoflavus(Gurtler et al 1994).

The natural role of antibiotics is subject to intensive debate. It has been argued that their main function lies in cell to cell communication(Davies 2006). However, antibiotics may well act as weapons, and bioactivity is influenced by social and competitive interactions between strains(Abrudan et al 2015). Interestingly, it is becoming evident that the small 
inorganic VCs such as hydrogen sulfide $\left(\mathrm{H}_{2} \mathrm{~S}\right)$ and nitric oxide (NO) play a major role in modulating antibiotic activity and resistance(Avalos et al 2018b). $\mathrm{H}_{2} \mathrm{~S}$ production protects bacteria against antibiotics targeting DNA, RNA, protein and cell wall biosynthesis(Shatalin et al 2011). Bacillus anthracis produces volatile NO to protect itself against oxidative stress and helps the bacterium to survive in macrophages, thus playing a key role in escaping the host defense(Gusarov and Nudler 2005, Shatalin et al 2008). However, production of NO also directly protects bacteria against a broad spectrum of antibiotics(Gusarov et al 2009, van Sorge et al 2013). Ammonia induced resistance to tetracycline, by increasing the level of polyamines which leads to a modification of membrane permeability(Bernier et al 2011). There is also some experimental evidence that suggests that VCs may affect membrane integrity(Fadli et al 2014, Yung et al 2016), which in turn may make the cells more susceptible to other cell-damaging compounds, such as antibiotics. The lack of information makes it hard to mimic the biological effect and more so to pinpoint the responsible molecules of such activity.

In this study we show for the first time that Streptomyces can produce surprisingly high levels of ammonia that affect surrounding bacteria even over long distances. The concentrations accumulated away from the colonies are so high that the ammonia acts as an antibiotic, inhibiting the growth of Escherichia coli and Bacillus subtilis. We also show that the production of ammonia in Streptomyces can be controlled by varying growth conditions, and depends on the glycine cleavage system. E. coli cells gain resistance against the ammonia by reducing the expression of the two-component system OmpR-EnvZ, thereby counteracting passage through the outer membrane porins. 
MATERIALS AND METHODS

Strains, media, culture conditions and antimicrobial assays.

Strains used in this study are listed in Table S1. The Streptomyces strains were grown on Soy Flour Mannitol (SFM) agar plates to prepare spore stocks. Escherichia coli strain AS19-RImA(Liu and Douthwaite 2002) and B. subtilis 168(Barbe et al 2009) were used as test microorganisms and grown on Luria-Bertani (LB) agar plates.

Volatile antimicrobial assays were performed using a petri dish with two compartments, one filled with SFM media for Streptomyces growth and the second one with LB +/- TES buffer 50-100 mM. Streptomyces strains were streaked on the SFM side and allowed to grow for 5 days after which, E. coli or B. subtilis were inoculated on the LB side using a concentration of $10^{4}$ and $10^{3} \mathrm{cfu} / \mathrm{mL}$ respectively.

\section{Collection and analysis of VCs.}

VCs produced by Streptomyces monocultures grown on SFM agar were collected using a glass petridish designed for trapping of the volatile headspace(Garbeva et al 2014). The lid of the glass petridish contains an outlet specially designed to hold a stainless steel column packed with 200 mg Tenax ${ }^{\circledR}$ TA 60/80 material (CAMSCO, Houston, TX, USA). Samples were taken in triplicates from day 3 to day 5 of growth; after that, the Tenax steel traps were sealed and stored at $4^{\circ} \mathrm{C}$ until GC-Q-TOF analysis.

Trapped volatiles were desorbed using an automated thermodesorption unit (model UnityTD-100, Markes International Ltd., United Kingdom) at $210^{\circ} \mathrm{C}$ for $12 \mathrm{~min}$ (Helium flow $50 \mathrm{ml} / \mathrm{min}$ ) and trapped on a cold trap at $-10^{\circ} \mathrm{C}$. The trapped volatiles were introduced into the GC-QTOF (model Agilent 7890B GC and the Agilent 7200A QTOF, USA) by heating the cold trap for $3 \mathrm{~min}$ to $280^{\circ} \mathrm{C}$. A $30 \times 0.25 \mathrm{~mm}$ ID RXI-5MS column with a film thickness of 0.25 
$\mu \mathrm{m}$ was used (Restek $13424-6850$, USA). Temperature program used was as follows: $39^{\circ} \mathrm{C}$ for

in MZmine. The files were imported to MZmine V2.14.2(Pluskal et al 2010) and compounds

were identified via their mass spectra using deconvolution function (Local-Maximum

algorithm) in combination with two mass-spectral-libraries: NIST 2014 V2.20 (National

with those found in the NIST and in the in-house NIOO-KNAW LRI database. After

deconvolution and mass identification peak lists containing the mass features of each

treatment (MZ-value/Retention time and the peak intensity) were created and exported as

CSV files for statistical processing via MetaboAnalyst V3.0 (www.metaboanalyst.ca; (Xia et al 2015)).

\section{pH change, ammonia determination and toxicity.}

122 Change in $\mathrm{pH}$ of the growth media was determined by the addition of phenol red indicator 
After 5 days, the ammonia was determined using the Quantofix ${ }^{\circledR}$ ammonium test kit.

127

128

129

Pictures were recorded to obtain a qualitative measurement of ammonia production from each strain.

Quantification of ammonia accumulation inside the LB agar was determined by extracting the liquid from the LB agar by centrifugation. For this, centrifuge tube filters were used (spin-X $X^{\circledR} 0.22 \mu \mathrm{m}$ cellulose acetate, Corning Inc. USA), $1 \mathrm{~cm}^{2}$ of agar was put inside the filter tube and centrifuge at 13,000 rpm for $20 \mathrm{~min}$. The eluate $(\sim 200 \mu \mathrm{L})$ was used to quantify the ammonium concentration in comparison to a standard curve. The standard curve was made with LB agar containing 0-50 mM concentrations of ammonia. Ammonia solution (25\% in $\mathrm{H}_{2} \mathrm{O}$, J.T. Baker 6051) was used as source of ammonia. The liquid was extracted from the agar the same way as described before and used together with the Quantofi $x^{\circledR}$ ammonium kit to obtain a semi-quantitative measure of ammonia accumulation inside the agar.

To determine the toxicity of ammonia, E. coli and B. subtilis were incubated in the automated Bioscreen C (Lab systems Helsinki, Finland) in the presence of increasing concentrations of ammonia. Each dilution was prepared in LB containing an inoculum of $10^{5} \mathrm{cfu} / \mathrm{mL}+$ different volumes of ammonia solution (J.T. Baker) to give the following final concentrations: $1,5,10,15,16,17,18,20,25,30,40$ and $50 \mathrm{mM}$. The final working volume in each well of the honeycomb was $100 \mu \mathrm{L}$. Cultures were incubated at $37^{\circ} \mathrm{C}$ overnight with continuous shaking. O.D. measurements (wideband) were taken every $30 \mathrm{~min}$ for $20 \mathrm{~h}$. The data and growth curves were calculated from triplicates.

\section{HCN determination.}



allowed to dry protected from light. The filter paper was placed next to Streptomyces pregrown for 2 days. Pseudomonas donghuensis P482 was used as positive control. Strains were incubated at $30^{\circ} \mathrm{C}$ until blue coloration of the filter paper was evident.

\section{Whole genome sequencing}

Genome sequencing of $E$. coli AS19-RImA- (Avalos et al 2018a) and its mutant ARM9 was performed using Illumina HiSEQ and PacBio RfavaS at Baseclear BV, Leiden (The Netherlands). Paired-end sequence reads were generated using the Illumina HiSeq 2000 system and mapping the individual reads against the reference genome of $E$. coli $B$ str. REL606. The contigs were placed into superscaffolds based on the alignment of the PacBio CLC reads. Alignment was performed with BLASR(Chaisson and Tesler 2012). Genome annotation was performed using the Baseclear annotation pipeline based on the Prokaryotic performed using the CLC genomics workbench version 6.5. The initial list of variants was filtered using the Phred quality score and false positives were reduced by setting the minimum variant frequency to $70 \%$ and the minimum number of reads that should cover a position was set to 10 . Relevant mutations were confirmed by PCR analysis. The genome of

170 E. coli AS19-RImA- has been published, with accession number CP027430 (Avalos et al 171 2018a). 


\section{Genetic complementation of ompR and envZ.}

E. coli strain AS19-RImA- suppressor mutant ARM9 was complemented by inserting the ompR or envZ genes in pCA24N from the ASKA collection(Kitagawa et al 2005). Cells of suppressor mutant ARM9 containing the plasmid were inoculated in LB + Chloramphenicol $(25 \mu \mathrm{g} / \mathrm{mL})$ with or without IPTG $0.1 \mathrm{mM}$ for induction of the gene expression.

\section{RNA sequencing}

For RNA extraction E. coli cells were grown to an O.D. 600 of 0.5, RNA Protect Bacteria Reagent (Qiagen Cat No. 76506) was added according to manufacturer instructions. Cells were pelleted and re-suspended in boiling $2 \%$ SDS +16 mM EDTA followed by extraction with Phenol:chloroform:Isoamyl alcohol (25:24:1) pH 6.6. (VWR Prolabo 436734C). Aqueous phase was precipitated with $3 \mathrm{M}$ sodium acetate $\mathrm{pH} 5.2$ and pure ethanol, washed with $70 \%$ Ethanol and re-suspended in RNAse-free water. DNA was removed using 5 units of DNAsel (Fermentas \#EN0521) with further purification using again phenol:chloroform:isoamyl alcohol and precipitation with sodium acetate and ethanol. The final pellet was dissolved in RNase free water.

RNA sequencing and analysis was performed by Baseclear BV (Leiden, The Netherlands). Ribosomal RNA was subsequently removed with a Ribo-Zero kit (Epicenter) and the remaining RNA used as input for the Illumina TruSeq RNA-seq library preparation. Once fragmented and converted into double strand cDNA, the fragments (about 100-200 bp) were ligated with DNA adapters at both ends and amplified via PCR. The resulting library was then sequenced using an Illumina Sequencer. The FASTQ sequence reads were generated using the Illumina Casava pipeline version 1.8.3. Initial quality assessment was based on data passing the Illumina Chastity filtering. Subsequently, reads containing adapters and/or PhiX 
control signals were removed using an in-house filtering protocol. The second quality assessment was based on the remaining reads using the FASTQC quality control tool version 0.10.0.

For the RNA-Seq analysis the quality of the FASTQ sequences was enhanced by trimming off low-quality bases using the "Trim sequences" option present in CLC Genomics Workbench Version 6.0.4 (QIAGEN, Bioinformatics). The quality-filtered sequence reads were used for further analysis with CLC Genomics Workbench. First an alignment against the reference and calculation of the transcript levels was performed using the "RNA-Seq" option. Subsequent comparison of transcript levels between strains and statistical analysis was done with the "Expression analysis" option, calculating so-called RPKM values. These are defined as the reads per kilobase per million mapped reads(Mortazavi et al 2008) and normalizes for the difference in the number of mapped reads between samples and for transcript length. The RNAseq data has been submitted to the GEO (Gene Expression Omnibus) from NCBI (National Biotechnology Center Information) with GEO accession number GSE111370.

\section{Synergism of Streptomyces AB-VCs with soluble antibiotics.}

Synergistic assays were performed using a petri dish with two compartments, one filled with SFM media for Streptomyces growth and the second one with LB. Streptomyces strains were streaked on the SFM side and allowed to grow for 5 days at $30^{\circ} \mathrm{C}$ after which, $E$. coli or $B$. subtilis were inoculated on the LB side using a culture grown to an O.D. $=0.5 .100 \mu \mathrm{L}$ were streaked on the LB side. Afterwards, $6 \mathrm{~mm}$ filter discs (Whatman ${ }^{\mathrm{TM}}$ ) were placed on top of the LB and $10 \mu \mathrm{L}$ of each antibiotic spotted on the filter disc. Two different concentrations per antibiotic were tested: ampicillin 500, $31 \mu \mathrm{g} / \mathrm{mL}$; erythromycin 250, $31 \mu \mathrm{g} / \mathrm{mL}$; kanamycin 1000, $500 \mu \mathrm{g} / \mathrm{mL}$; tylosin 500, $62 \mu \mathrm{g} / \mathrm{mL}$; actinomycin 500, $62 \mu \mathrm{g} / \mathrm{mL}$; 
spectinomycin $1000,500 \mu \mathrm{g} / \mathrm{mL}$; streptomycin $500,62 \mu \mathrm{g} / \mathrm{mL}$. Plates were incubated at $37^{\circ} \mathrm{C}$ overnight and pictures recorded after $20 \mathrm{~h}$ of growth.

\section{RESULTS AND DISCUSSION}

\section{VCs as bioactive agents in long-distance antibiosis}

Since streptomycetes actively produce antifungal VCs, we set out to investigate if also antibacterial VCs could be produced by these bacteria. After all, this would give them a competitive advantage by inhibiting other bacteria at longer distances. To investigate this, streptomycetes were grown physically separated from indicator/target bacteria by a polystyrene barrier. Air-borne VCs can pass over the barrier, but it does not allow passage of canonical (soluble) antibiotics. As indicator strains we used Bacillus subtilis and Escherichia coli strain AS19-RImA- (referred to as E. coli ASD19 from now on). The latter has known antibiotic sensitivity(Liu and Douthwaite 2002). Interestingly, the streptomycetes showed varying volatile activity against $E$. coli ASD19; the indicator strain failed to grow adjacent to Streptomyces sp. MBT11 or S. venezuelae, but grew normally next to S. coelicolor, S. lividans and S. griseus (Figure 1A). B. subtilis was not inhibited by any of the former strains.

We then wanted to assess whether the production of antimicrobial volatile compounds (AMVCs) could be elicited by varying the growth conditions. We previously showed that growth at $\mathrm{pH} 10, \mathrm{~N}$-acetylglucosamine, starch or yeast extract pleiotropically enhanced the production of antibiotics in many Streptomyces species(Zhu et al 2014). Interestingly, in contrast to a neutral $\mathrm{pH}$, when glycine/ $\mathrm{NaOH}$ buffer was added to raise the $\mathrm{pH}$ to $10, S$. griseus produced VCs that completely inhibited growth of $E$. coli and B. subtilis 
(Figure 1B). AMVC production by Streptomyces species MBT11 was also enhanced by growth under any of the conditions tested (Table S2). ideal system to elucidate the nature of the bioactive molecules by statistical methods. Correlation between bioactivity and metabolic profiles allows efficient reduction of candidate molecules (Gubbens et al 2014, Wu et al 2015). Therefore, GC-Q-TOF-based metabolomics was performed to compare the VC profiles of S. coelicolor, Streptomyces sp. MBT11, S. venezuelae and S. griseus (the latter grown with and without glycine buffer $\mathrm{pH}$ 10). Despite the antimicrobial activity, no volatile organic compound (VOC) was detected that correlated statistically to the bioactivity, nor did we see any significant difference between the metabolome profiles of $S$. griseus grown with or without the glycine buffer methylisoborneol (2-MIB) and 2-methylenebornane by the active strains compared to the non-active strains. However, mutants of S. griseus that lacked either or even all of the terpene cyclases, still retained their volatile antibacterial activity (data not shown).

\section{The main inhibitory molecule is ammonia}

VCs may induce a change in pH away from colonies(Jones et al 2017, Letoffe et al 2014), and we therefore wondered whether the VC bioactivity was accompanied by a $\mathrm{pH}$ change on the receiver side. To assess this, we used phenol red, which changes from pale orange to bright pink when the media becomes alkaline. Interestingly, a gradual increase was seen in the $\mathrm{pH}$ 
the Streptomyces biomass, and after 5 days the receiver side had turned completely pink (Figure 2A). At that point, the $\mathrm{pH}$ had increased to around 8.5. The $\mathrm{pH}$ increase correlated fully to the antibiosis, with growth inhibition of E. coli close to the Streptomyces biomass after 3 days, while after 5 days the growth of E. coli was fully inhibited (Figure 2B). Further in support of $\mathrm{pH}$-dependent growth inhibition, E. coli grew normally when the media was buffered with $50 \mathrm{mM}$ TES (pH 7). However, the $\mathrm{pH}$ itself was not the cause of the inhibition, since the E. coli cells grew apparently normal on media adjusted to $\mathrm{pH} 9$ (Figure 2C). Also, we previously showed that even at $\mathrm{pH} 10$ antibiotic susceptibility is similar to growth at $\mathrm{pH}$ 7(Zhu et al 2014).

Ammonia and trimethylamine are VCs known to induce a pH change(Bernier et al 2011, Čepl JJ 2010, Jones et al 2017, Letoffe et al 2014). We also tested production of hydrogen cyanide $(\mathrm{HCN})$, a known AMVC produced by, among others, rhizospheric streptomycetes(Anwar et al 2016); however, none of the strains produced detectable amounts of HCN (Figure S1). Furthermore, under our growth conditions, TMA was not detected in the headspace of the Streptomyces strains (Figure S2). Ammonia production was determined using the Quantofix ${ }^{\circledast}$ Ammonium detection kit. Interestingly, antibiosis by the streptomycetes fully correlated to an increase in ammonia production (Figure 2D). To determine how much ammonia was accumulated, the Streptomyces strains were grown for 5 days, and the agar on the receiver side extracted. Agar containing different concentrations of ammonia was used to create a standard curve. S. coelicolor, S. lividans and S. griseus (pH 7) accumulated only 2-5 mM ammonia, while the growth-inhibiting Streptomyces sp. MBT11, S. venezuelae and S. griseus (the latter only with added $\mathrm{Gly} / \mathrm{NaOH}$ buffer $\mathrm{pH} 10$ ) had accumulated between 15-30 mM ammonia (Figure 3A). 


\section{Ammonia is derived from glycine cleavage}

We then wondered if ammonia was generated from glycine metabolism, because a

is the glycine cleavage system (GCV) that converts glycine into $\mathrm{CO}_{2}$, ammonia and a methylene group that is transferred to tetrahydrofolate (THF) to form $\mathrm{N}_{5}, \mathrm{~N}_{10}$-methyleneTHF(Kikuchi et al 2008, Tezuka and Ohnishi 2014). Importantly, when S. griseus was grown on SFM agar containing just glycine at concentrations as low as $0.1 \%(w / v)$, this time without 4A). We then also grew S. coelicolor on SFM agar with increasing concentrations of glycine. Interestingly, at concentrations of $1 \%(\mathrm{w} / \mathrm{v})$ glycine or higher, also $S$. coelicolor fully inhibited the indicator cells. This suggests that at sufficiently high concentrations of glycine, all streptomycetes may produce so much ammonia that it inhibits the growth of other bacteria. We then tested the direct involvement of the GCV system(Tezuka and Ohnishi 2014), which consists of three enzymes (GcvL, GcvP, GcvT) and a carrier protein: GcvH (Figure 4B). and Ohnishi 2014) were unable to produce ihibiting amounts of ammonia, even when grown on high amounts of glycine. A mutant of S. griseus lacking the 5'UTR of gcvP (Tezuka and Ohnishi 2014) still produced sufficient ammonia to inhibit the growth of $E$. coli cells, but this 
suggests that in both S. coelicolor and S. griseus, volatile ammonia is primarily derived from

317

318

319 the GCV system, and as expected, GcvT is the key enzyme responsible for the production of ammonia from glycine. Inactivation of $g c v P$ is also sufficient to block volatile ammonia production in S. coelicolor, fully in line with the idea that the key system is GCV, while in $S$. griseus GcvP can be by-passed by another (yet unknown) enzyme.

A major difference between S. coelicolor and S. griseus on the one had, and $S$. venezuelae and Streptomyces sp. MBT11 on the other, is that the latter two strains do not require any added glycine to produce levels of ammonia above the MIC. Ammonia may be derived from various metabolic enzymes, such as ammonia lyases, deaminases, deiminases and pyridoxamine phosphate oxidases. We are currently performing a large-scale phylogenomics and mutational analysis to identify the gene(s) that are responsible for the overproduction of ammonia in these strains.

\section{OmpR is key to ammonia resistance}

To obtain more insights into the cellular response of $E$. coli cells to ammonia, we selected for spontaneous ammonia-resistant mutants. After two days of growth next to Streptomyces sp. MBT11, several colonies appeared that were able to withstand the accumulated ammonia and likely had sustained one or more suppressor mutations. Four of these colonies were analyzed further, which showed different levels of resistance as indicated by the colony size (Figure S3A). Of these, suppressor mutant ARM9 was selected for its high resistance (Figure 5A left). Strain ARM9 was reproducibly more resistant to ammonia than its parent, with MICs of $25 \mathrm{mM}$ and $20 \mathrm{mM}$, respectively. This is a highly significant difference, as we previously showed that $20 \mathrm{mM}$ is precisely the tipping point for ammonia sensitivity of $E$. coli (Figure $5 B)$. 
include high concentrations of ammonia, therefore we hypothesized that a mechanism other

the nature of the mutation(s) sustained by ARM9, its genome sequence was compared to

that of its parent E. coli ASD19 (Table S3). In total 658 mutations were found by single

nucleotide permutation (SNP) analysis, of which 198 gave rise to amino acid changes or

insertions or deletions. However, one change immediately stood out, namely the

introduction of two insertion elements (insA_31 and insB_31) in-between the -35 and -10

consensus sequences of the promoter for ompR-envZ, which encode the two-component

right). This TCS is involved in osmoregulation in response to environmental signals(Nikaido

2003) and regulates the expression of outer membrane porins OmpF and OmpC.

Importantly, these are known to be involved in antibiotic resistance regulated by osmotic pressure and $\mathrm{pH}($ Fernandez and Hancock 2012), and to reduce the responsiveness of $E$. coli cells to VCs(Kim et al 2013).

\section{Reduced transcription of the ompR-envZ operon is the cause of ammonia resistance}

Considering the location right in the middle of the promoter, we expected that the IS elements in the ompR-envZ promoter reduced the transcription of these crucial TCS genes. To establish the transcriptional consequences of the IS insertion into the ompR-envZ promoter region, RNAseq was performed on E. coli ASD19 and its suppressor mutant ARM9

362 grown in LB media until mid-exponential phase $\left(\mathrm{OD}_{600} 0.5\right)$, and the global transcription 
regulated as a result of a clustering analysis using a cut-off value of a fold change +/- 2.0.

365

These data confirm the downregulation of ompR and envZ genes and other related genetic elements like omrA, a small mRNA that negatively regulates ompR expression. Additionally, genes involved in amino-acid metabolism were down regulated, including the astABCE gene cluster involved in the ammonia-producing arginine catabolic pathway, aspA that is involved in the conversion of L- aspartate into fumarate and ammonia, and thaC for catabolism of tryptophan, which again releases ammonia.

To confirm that indeed the reduced transcription of ompR-envZ was the major cause for the acquired ammonia resistance, $E$. coli mutant ARM9 was genetically complemented by the introduction of constructs from the ASKA collection(Kitagawa et al 2005) expressing either ompR or envZ. Introduction of constructs expressing either ompR or envZ restored ammonia sensitivity, while transformants harboring the empty plasmid continued to be resistant (Figure $5 \mathrm{C}$ ). This strongly suggests that the reduced expression of ompR and envZ was the sole cause of the acquired ammonia resistance. It is important to note that mutant ARM9 had also become resistant to AMVCs produced by S. venezuelae and by S. griseus (the latter grown on glycine), again providing evidence that all strains act by producing ammonia as the AMVC (Figure S3B).

Taken together, these data show that E. coli responds to exposure to ammonia by reducing ompR-envZ transcription, down regulating the expression of OMPs to minimize the passage of small molecules, and by the reduction of ammonia biosynthesis. Both responses are aimed at defense against the accumulation of toxic levels of ammonia. When exposed to ammonia, E. coli ompR mutants were shown to be significantly more sensitive to tetracycline than the parental strain (Bernier et al 2011). Our results show that reducing the expression of OMPs is a defense mechanism against ammonia toxicity extending also earlier 
observations that ompF mutants show impaired response to VOCs that affect the motility of

E. coli(Kim et al 2013).

\section{Ammonia released by Streptomyces modifies sensitivity to canonical antibiotics.}

Since ammonia is an AMVC that can reach far from the colony, we considered that the molecule may play a role in long-distance competition with other microbes in the soil, e.g. by modifying the effect of other antibiotics produced by actinomycetes, such as erythromycin, kanamycin, actinomycin, spectinomycin and streptomycin. This could be an interesting synergistic effect whereby weapons produced by the strain itself are potentiating via

Thus, bioactive VCs from Streptomyces modulate the activity of soluble antibiotics at longer microbial communities.

implications on ecology and antibiotic activity.

410 Streptomyces VCs have a perceivable impact on the $\mathrm{pH}$ of their surroundings. Research has

411 shown that richness and diversity of bacterial soil microbiomes is largely explained by the 
soil $\mathrm{pH}$ (Fierer and Jackson 2006) with acidic soils having the lowest diversity. Basic environments favor bacterial growth while acidic environments do it for fungi(BárcenasMoreno et al 2011, Rousk et al 2009). Studies have shown that VCs are more strongly adsorbed in alkaline soils, especially those containing a high organic carbon content (2.9\%)(Serrano and Gallego 2006). The release of ammonia by bacteria could have several major implications. First of all, the volatile characteristic allows it to travel far from the producer mediating long-distance interactions. To the best of our knowledge this is the first report showing that soil bacteria such as Streptomyces actively kill other bacteria through the air, via the production of ammonia. Additional ecological impact is provided by work of others that shows that ammonia produced by actinobacteria and in particular Streptomyces acts as a plant-growth promoter(Passari et al 2017). We hypothesize that such plant-growth promotion may at, least in part, be due to protection against plant pathogenic microbes and in return expand the 'living room' of the volatile-producing Streptomyces.

Finally, the release of ammonia could help the solubility and diffusion of other types of secondary metabolites, while sensitizing competing bacteria, therefore, the production of a small low-cost ammonia is also a logical strategy to enhance the activity of more complex and hence costly antibiotics, such as polyketides, non-ribosomal peptides or $\beta$-lactams. After all, synthesis of these compounds requires expensive high-energy precursors like ATP, NADPH and acyl-CoAs. This is applicable both to antibiotics produced by the organism itself, and to those produced by bacteria further away from the colony. The validity of this concept of "antibiotic piracy" requires further experimental testing.

In conclusion, our work shows that several streptomycetes use ammonia as a lowcost airborne weapon to change their surrounding environment, thereby making their own more costly defense mechanism more effective. In this microbial warfare, the surrounding 
bacteria then respond by reducing the permeability of their outer membrane and by may offer new opportunities for agricultural and/or medical applications, such as for crop protection, plant-growth promotion, and antimicrobial compounds and to continue to understand the role of these molecules in microbial interactions.

\section{ACKNOWLEDGEMENTS}

This work was supported by Grant No. 313599 from The Mexican National Council of Science and Technology (CONACYT) to MA, by VIDI grant 864.11.015 from the Netherlands Organization for Scientific Research (NWO) to PG and by grant 14221 from the Netherlands

$450 \quad \mathrm{R} / \mathrm{mA} \mathbf{A}^{-}$

453 The authors declare no competing financial interests. 


\section{REFERENCES}

Abrudan MI, Smakman F, Grimbergen AJ, Westhoff S, Miller EL, van Wezel GP et al (2015). Socially mediated induction and suppression of antibiosis during bacterial coexistence. Proc Natl Acad Sci U S A 112: 11054-11059.

Anwar S, Ali B, Sajid I (2016). Screening of Rhizospheric Actinomycetes for Various In-vitro and In-vivo Plant Growth Promoting (PGP) Traits and for Agroactive Compounds. Front Microbiol 7: 1334.

Audrain B, Farag MA, Ryu CM, Ghigo JM (2015). Role of bacterial volatile compounds in bacterial biology. FEMS Microbiol Rev 39: 222-233.

Avalos M, Boetzer M, Pirovano W, Arenas NE, Douthwaite S, van Wezel GP (2018a). Complete Genome Sequence of Escherichia coli AS19, an Antibiotic-Sensitive Variant of E. coli Strain B REL606. Genome Announc 6: e00385-00318.

Avalos M, van Wezel GP, Raaijmakers JM, Garbeva P (2018b). Healthy scents: microbial volatiles as new frontier in antibiotic research? Curr Opin Microbiol 45: 84-91.

Barbe V, Cruveiller S, Kunst F, Lenoble P, Meurice G, Sekowska A et al (2009). From a consortium sequence to a unified sequence: the Bacillus subtilis 168 reference genome a decade later. Microbiology (Reading, England) 155: 1758-1775.

Bárcenas-Moreno G, Rousk J, Bååth E (2011). Fungal and bacterial recolonisation of acid and alkaline forest soils following artificial heat treatments. Soil Biol Biochem 43: 1023-1033.

Barka EA, Vatsa P, Sanchez L, Gaveau-Vaillant N, Jacquard C, Klenk HP et al (2016). Taxonomy, Physiology, and Natural Products of Actinobacteria. Microbiol Mol Biol Rev 80: 1-43.

Bérdy J (2012). Thoughts and facts about antibiotics: where we are now and where we are heading. J Antibiot (Tokyo) 65: 385-395.

Bernier SP, Letoffe S, Delepierre M, Ghigo JM (2011). Biogenic ammonia modifies antibiotic resistance at a distance in physically separated bacteria. Mol Microbiol 81: 705-716.

Castric KF, Castric PA (1983). Method for rapid detection of cyanogenic bacteria. Appl Environ Microbiol 45: 701-702.

Čepl JJ PI, Blahǔšková A, Cvrčková F, Markoš A. (2010). Patterning of mutually interacting bacterial bodies: close contacts and airborne signals.pdf. BMC Microbiol 10.

Chaisson MJ, Tesler G (2012). Mapping single molecule sequencing reads using basic local alignment with successive refinement (BLASR): application and theory. BMC Bioinformatics 13: 238. 
Citron CA, Barra L, Wink J, Dickschat JS (2015). Volatiles from nineteen recently genome sequenced actinomycetes. Org Biomol Chem 13: 2673-2683.

Conroy MJ, Durand A, Lupo D, Li X-D, Bullough PA, Winkler FK et al (2007). The crystal structure of the Escherichia coli AmtB-GInK complex reveals how GInK regulates the ammonia channel. Proc Natl Acad Sci U S A 104: 1213.

Cordovez V, Carrion VJ, Etalo DW, Mumm R, Zhu H, van Wezel GP et al (2015). Diversity and functions of volatile organic compounds produced by Streptomyces from a disease-suppressive soil. Front Microbiol 6: 1081.

Davies J (2006). Are antibiotics naturally antibiotics? J Ind Microbiol Biotechnol 33: 496-499.

Fadli M, Chevalier J, Hassani L, Mezrioui NE, Pages JM (2014). Natural extracts stimulate membraneassociated mechanisms of resistance in Gram-negative bacteria. Lett Appl Microbiol 58: 472-477.

Fernandez L, Hancock RE (2012). Adaptive and mutational resistance: role of porins and efflux pumps in drug resistance. Clin Microbiol Rev 25: 661-681.

Fierer N, Jackson RB (2006). The diversity and biogeography of soil bacterial communities. Proc Natl Acad Sci U S A 103: 626-631.

Garbeva P, Hordijk C, Gerards S, de Boer W (2014). Volatile-mediated interactions between phylogenetically different soil bacteria. Front Microbiol 5: 289.

Gubbens J, Zhu H, Girard G, Song L, Florea BI, Aston P et al (2014). Natural product proteomining, a quantitative proteomics platform, allows rapid discovery of biosynthetic gene clusters for different classes of natural products. Chem Biol 21: 707-718.

Gurtler H, Pedersen R, Anthoni U, Christophersen C, Nielsen PH, Wellington EM et al (1994). Albaflavenone, a sesquiterpene ketone with a zizaene skeleton produced by a streptomycete with a new rope morphology. J Antibiot (Tokyo) 47: 434-439.

Gusarov I, Nudler E (2005). NO-mediated cytoprotection: instant adaptation to oxidative stress in bacteria. Proc Natl Acad Sci U S A 102: 13855-13860.

Gusarov I, Shatalin K, Starodubtseva M, Nudler E (2009). Endogenous nitric oxide protects bacteria against a wide spectrum of antibiotics. Science 325: 1380-1384.

Hopwood DA (2007a). Streptomyces in Nature and Medicine. The Antibiotic Makers. Oxford University Press Inc: New York, NY. 
Hopwood DA (2007b). Streptomyces in nature and medicine: the antibiotic makers. Oxford University Press: New York.

Jones SE, Ho L, Rees CA, Hill JE, Nodwell JR, Elliot MA (2017). Streptomyces exploration is triggered by fungal interactions and volatile signals. Elife 6 .

Kai M, Haustein M, Molina F, Petri A, Scholz B, Piechulla B (2009). Bacterial volatiles and their action potential. Appl Microbiol Biotechnol 81: 1001-1012.

Kikuchi G, Motokawa Y, Yoshida T, Hiraga K (2008). Glycine cleavage system: reaction mechanism, physiological significance, and hyperglycinemia. Proc Japan Acad Series B 84: 246-263.

Kim KS, Lee S, Ryu CM (2013). Interspecific bacterial sensing through airborne signals modulates locomotion and drug resistance. Nat Commun 4: 1809.

Kitagawa M, Ara T, Arifuzzaman M, loka-Nakamichi T, Inamoto E, Toyonaga $\mathrm{H}$ et al (2005). Complete set of ORF clones of Escherichia coli ASKA library (a complete set of E. coli K-12 ORF archive): unique resources for biological research. DNA Res 12: 291-299.

Letoffe S, Audrain B, Bernier SP, Delepierre M, Ghigo JM (2014). Aerial exposure to the bacterial volatile compound trimethylamine modifies antibiotic resistance of physically separated bacteria by raising culture medium pH. MBio 5: e00944-00913.

Liu M, Douthwaite S (2002). Activity of the ketolide telithromycin is refractory to Erm monomethylation of bacterial rRNA. Antimicrob Agents Chemother 46: 1629-1633.

Mortazavi A, Williams BA, McCue K, Schaeffer L, Wold B (2008). Mapping and quantifying mammalian transcriptomes by RNA-Seq. Nature methods 5: 621-628.

Nijland R, Burgess JG (2010). Bacterial olfaction. Biotechnol J 5: 974-977.

Nikaido H (2003). Molecular basis of bacterial outer membrane permeability revisited. Microbiol Mol Biol Rev 67: 593-656.

Passari AK, Mishra VK, Singh G, Singh P, Kumar B, Gupta VK et al (2017). Insights into the functionality of endophytic actinobacteria with a focus on their biosynthetic potential and secondary metabolites production. Scientific Reports 7: 11809.

Pluskal T, Castillo S, Villar-Briones A, Oresic M (2010). MZmine 2: Modular framework for processing, visualizing, and analyzing mass spectrometry-based molecular profile data. BMC Bioinformatics 11: 11. 
Que YA, Hazan R, Strobel B, Maura D, He J, Kesarwani M et al (2013). A quorum sensing small volatile molecule promotes antibiotic tolerance in bacteria. PLoS One 8: e80140.

Rousk J, Brookes PC, Bååth E (2009). Contrasting Soil pH Effects on Fungal and Bacterial Growth Suggest Functional Redundancy in Carbon Mineralization. Appl Env Microbiol 75: 1589-1596.

Schmidt R, Cordovez V, de Boer W, Raaijmakers J, Garbeva P (2015). Volatile affairs in microbial interactions. ISME J 9: 2329-2335.

Schöller CEG, Gürtler H, Pedersen R, Molin S, Wilkins K (2002). Volatile Metabolites from Actinomycetes. Journal of Agricultural and Food Chemistry 50: 2615-2621.

Schulz-Bohm K, Martín-Sánchez L, Garbeva P (2017). Microbial Volatiles: Small Molecules with an Important Role in Intra- and Inter-Kingdom Interactions. Frontiers in Microbiology 8.

Schulz S, Dickschat JS (2007). Bacterial volatiles: the smell of small organisms. Nat Prod Rep 24: 814842.

Serrano A, Gallego M (2006). Sorption study of 25 volatile organic compounds in several Mediterranean soils using headspace-gas chromatography-mass spectrometry. J Chromatography $A$ 1118: $261-270$.

Shatalin K, Gusarov I, Avetissova E, Shatalina Y, McQuade LE, Lippard SJ et al (2008). Bacillus anthracis-derived nitric oxide is essential for pathogen virulence and survival in macrophages. Proc Natl Acad Sci U S A 105: 1009-1013.

Shatalin K, Shatalina E, Mironov A, Nudler E (2011). H2S: a universal defense against antibiotics in bacteria. Science 334: 986-990.

Tezuka T, Ohnishi Y (2014). Two glycine riboswitches activate the glycine cleavage system essential for glycine detoxification in Streptomyces griseus. J Bacteriol 196: 1369-1376.

van Sorge NM, Beasley FC, Gusarov I, Gonzalez DJ, von Kockritz-Blickwede M, Anik S et al (2013). Methicillin-resistant Staphylococcus aureus bacterial nitric-oxide synthase affects antibiotic sensitivity and skin abscess development. J Biol Chem 288: 6417-6426.

Wang C, Wang Z, Qiao X, Li Z, Li F, Chen M et al (2013). Antifungal activity of volatile organic compounds from Streptomyces alboflavus TD-1. FEMS Microbiol Lett 341: 45-51.

Wirén Nv, Merrick M (2004). Regulation and function of ammonium carriers in bacteria, fungi, and plants. Molecular Mechanisms Controlling Transmembrane Transport. Springer Berlin Heidelberg: Berlin, Heidelberg. pp 95-120. 
625

631

632

633

634

635

636

637

638

639

640

641

642

643

644

645

646

647

648

649

650

651
Wu C, Kim HK, van Wezel GP, Choi YH (2015). Metabolomics in the natural products field - a gateway to novel antibiotics. Drug Discov Today Technol 13: 11-17.

Xia J, Sinelnikov IV, Han B, Wishart DS (2015). MetaboAnalyst 3.0-making metabolomics more meaningful. Nucleic Acids Res 43: W251-W257.

Yung PY, Grasso LL, Mohidin AF, Acerbi E, Hinks J, Seviour T et al (2016). Global transcriptomic responses of Escherichia coli K-12 to volatile organic compounds. Sci Rep 6: 19899.

Zhang L (2015). Identification and characterization of developmental genes in Streptomyces. PhD thesis, Leiden University, Leiden.

Zhu H, Swierstra J, Wu C, Girard G, Choi YH, van Wamel W et al (2014). Eliciting antibiotics active against the ESKAPE pathogens in a collection of actinomycetes isolated from mountain soils. Microbiology 160: 1714-1725. 


\section{TABLES}

Table 1. Clustering of the differentially down/up-regulated genes in ARM9 compared to ASD19. Only fold

\begin{tabular}{|c|c|c|c|}
\hline Cluster & Gene & Function & $\begin{array}{l}\text { Fold } \\
\text { Change }\end{array}$ \\
\hline \multicolumn{4}{|c|}{ DOWN-REGULATED } \\
\hline \multirow{10}{*}{$\begin{array}{l}\text { Membrane } \\
\text { Function/ } \\
\text { Transport }\end{array}$} & envZ & $\begin{array}{l}\text { sensory histidine kinase in two-component regulatory } \\
\text { system with OmpR }\end{array}$ & -16.43 \\
\hline & omrA & small regulatory RNA & -15.86 \\
\hline & yhdV & putative outer membrane protein & -15.86 \\
\hline & $o m p R$ & $\begin{array}{l}\text { response regulator in two-component regulatory system } \\
\text { with EnvZ }\end{array}$ & -14.84 \\
\hline & $d a c D$ & $\begin{array}{l}\text { D-alanyl-D-alanine carboxypeptidase, penicillin-binding } \\
\text { protein } 6 b\end{array}$ & -6.47 \\
\hline & $y q h H$ & outer membrane lipoprotein, Lpp paralog & -6.20 \\
\hline & ydiM & putative MFS transporter, membrane protein & -4.83 \\
\hline & yiaD & $\begin{array}{l}\text { multicopy suppressor of BamB, outer membrane } \\
\text { lipoprotein }\end{array}$ & -4.54 \\
\hline & $y a j R$ & putative transporter & -4.37 \\
\hline & $y h f L$ & small lipoprotein & -3.22 \\
\hline \multirow{4}{*}{$\begin{array}{l}\text { Domain: } \\
\text { EAL }\end{array}$} & bluF & $\begin{array}{l}\text { anti-repressor for YcgE, blue light-responsive, FAD-binding, } \\
\text { inactive c-di-GMP phosphodiesterase-like EAL domain } \\
\text { protein }\end{array}$ & -2.55 \\
\hline & yhjH & cyclic-di-GMP phosphodiesterase, FlhDC-regulated & -2.13 \\
\hline & $y c g G$ & $\begin{array}{l}\text { putative membrane-anchored cyclic-di-GMP } \\
\text { phosphodiesterase }\end{array}$ & -2.36 \\
\hline & ylie & $\begin{array}{l}\text { putative membrane-anchored cyclic-di-GMP } \\
\text { phosphodiesterase }\end{array}$ & -2.04 \\
\hline \multirow{16}{*}{$\begin{array}{l}\text { Aminoacyl- } \\
\text { tRNA } \\
\text { biosynthesis }\end{array}$} & alaw & tRNA-Ala & -2.02 \\
\hline & alax & tRNA-Ala & -2.30 \\
\hline & $\arg X$ & tRNA-Arg & -2.19 \\
\hline & $a s n V$ & tRNA-Asn & -2.76 \\
\hline & asnW & tRNA-Asn & -2.12 \\
\hline & $g \ln V$ & tRNA-GIn & -2.18 \\
\hline & $g \ln x$ & tRNA-GIn & -2.35 \\
\hline & hisR & tRNA-His & -2.03 \\
\hline & leuP & tRNA-Leu & -2.37 \\
\hline & leuT & tRNA-Leu & -3.34 \\
\hline & leuU & tRNA-Leu & -2.43 \\
\hline & pheV & tRNA-Phe & -3.56 \\
\hline & proK & tRNA-Pro & -2.24 \\
\hline & prol & tRNA-Pro & -2.94 \\
\hline & proM & tRNA-Pro & -2.76 \\
\hline & selc & tRNA-Sec & -2.07 \\
\hline
\end{tabular}




\begin{tabular}{|c|c|c|c|}
\hline & valV & tRNA-Val & -3.52 \\
\hline \multirow{6}{*}{$\begin{array}{l}\text { Aminoacid } \\
\text { metabolism }\end{array}$} & astA & arginine succinyltransferase & -2.17 \\
\hline & astB & succinylarginine dihydrolase & -2.09 \\
\hline & astC & succinylornithine transaminase, PLP-dependent & -2.13 \\
\hline & astE & succinylglutamate desuccinylase & -2.14 \\
\hline & feaR & transcriptional activator for $\operatorname{tyn} A$ and $f e a B$ & -2.02 \\
\hline & tnaC & tryptophanase leader peptide & -2.21 \\
\hline \multirow{4}{*}{$\begin{array}{l}\text { Fatty acid } \\
\text { Oxidation }\end{array}$} & fadA & 3-ketoacyl-CoA thiolase (thiolase I) & -2.20 \\
\hline & $f a d B$ & fatty acid oxidation complex, $\alpha$ component & -2.19 \\
\hline & fadH & 2,4-dienoyl-CoA reductase, NADH and FMN-linked & -2.14 \\
\hline & $\operatorname{prpB}$ & 2-methylisocitrate lyase & -2.07 \\
\hline \multicolumn{4}{|c|}{ UP-REGULATED } \\
\hline \multirow{2}{*}{ Prophage } & xis $D$ & pseudogene, exisionase in defective prophage DLP12 & 7.252 \\
\hline & $y / c l$ & DUF3950 family protein, DLP12 prophage & 5.318 \\
\hline \multirow{6}{*}{ Ribosome } & $\operatorname{rrsC}$ & $16 \mathrm{~S}$ ribosomal RNA & 3.93 \\
\hline & $r r f B$ & & 3.32 \\
\hline & $r r f C$ & & 2.18 \\
\hline & $r r f D$ & $5 S$ ribosomal RNA & 5.08 \\
\hline & $r r f G$ & & 3.55 \\
\hline & $r r f H$ & & 3.38 \\
\hline \multirow{4}{*}{ Pilus } & $\operatorname{fim} C$ & periplasmic chaperone & 2.42 \\
\hline & $\operatorname{fim} F$ & minor component of type 1 fimbriae & 2.05 \\
\hline & $p p d D$ & putative prepilin peptidase-dependent pilin & 4.35 \\
\hline & $y d e R$ & putative fimbrial-like adhesin protein & 2.18 \\
\hline
\end{tabular}


A
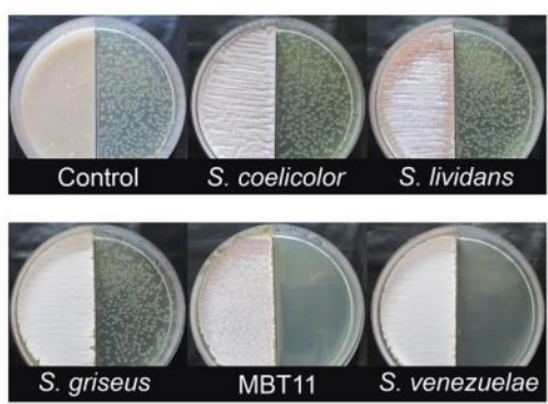

C

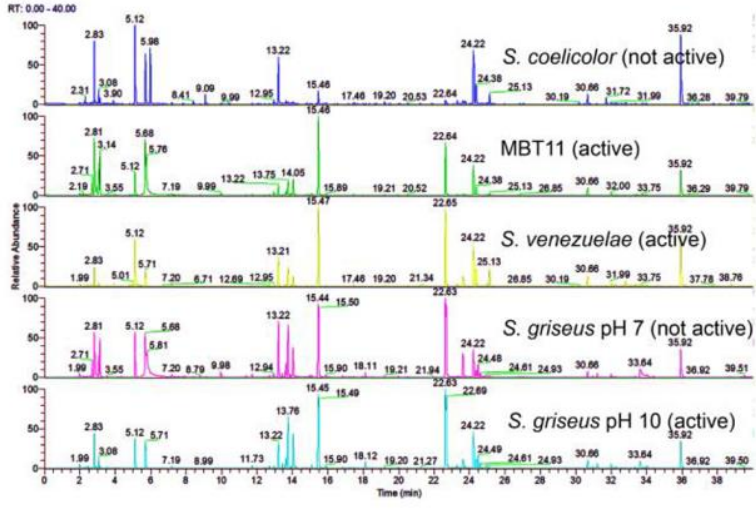

B
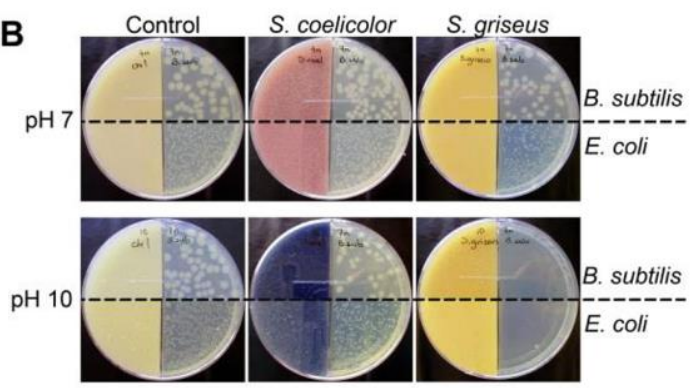

D

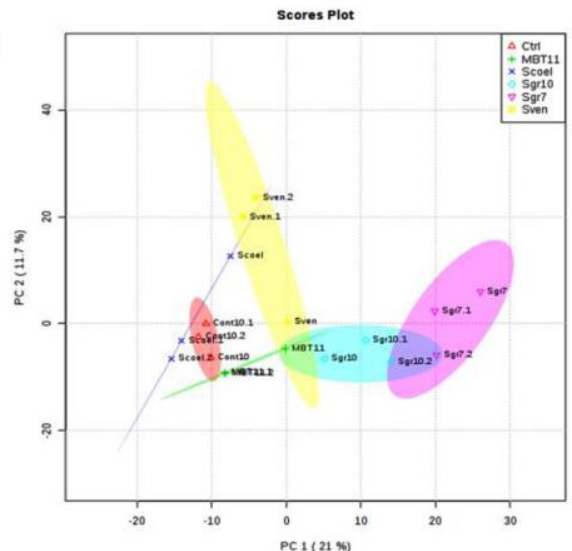

670

671 Figure 1. Bioactivity and metabolomic analysis of VCs released by streptomycetes. A. Bioactivity of VCS

672 released by selected Streptomyces strains against E. coli strain ASD19. B. Volatile antibiotic activity of different

673 Streptomyces strains grown at $\mathrm{pH} 7$ or $\mathrm{pH} 10$, the latter by addition of a glycine/NaOH buffer; E. coli strain ASD19 was the indicator strain. C. Comparison of GC-chromatogram of VCs from bioactive and non-bioactive 

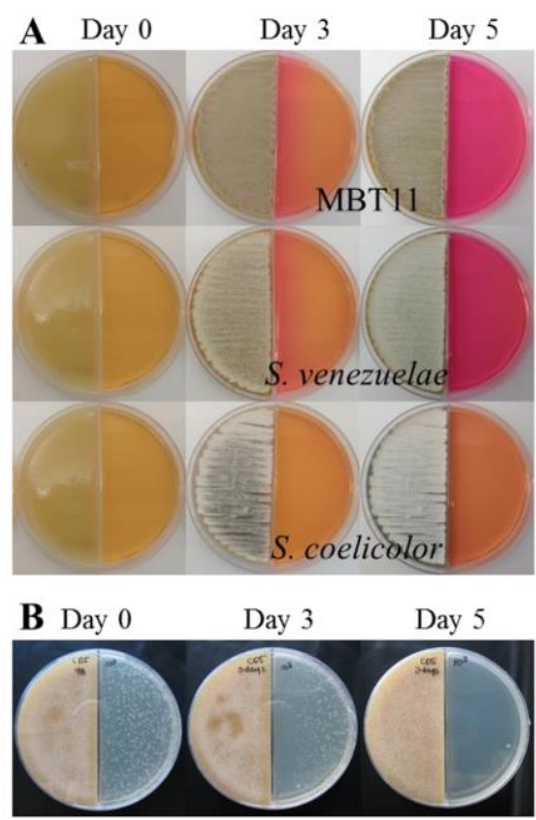

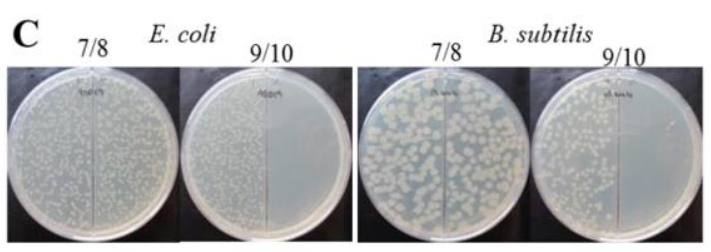

D

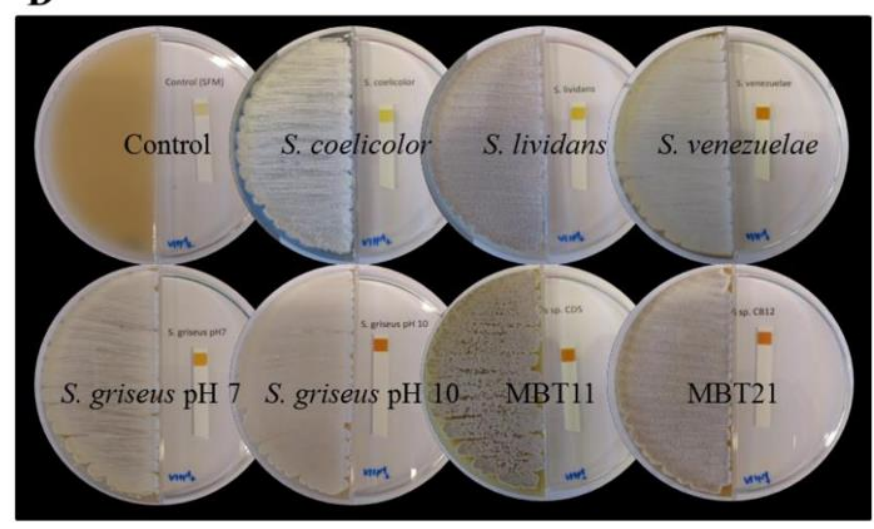

Figure 2. $\mathrm{pH}$ increase is caused by high ammonia production. A. $\mathrm{pH}$ change illustrated by the color change of are estimated according to the color chart indicator from the Quantofix ${ }^{\circledR}$ ammonium detection Kit.

the indicator (Phenol red $0.002 \%$ ). LB medium alkalization after 3 and 5 days of growth of Streptomyces sp.

MBT11 and S. venezuelae, no alkalization was caused by VCs produced by S. coelicolor. B. Streptomyces sp. MBT11 antimicrobial VCs production curve against E. coli strain ASD19. C. E. coli strain ASD19 and B. subtilis growth under different $\mathrm{pH}$ adjusted with glycine/NaOH buffer. $\mathbf{D}$. $\mathrm{NH}_{3}$ emission. Test strips on the right compartment show the production of $\mathrm{NH}_{3}$ by Streptomyces strains. S. coelicolor and S. lividans ( 10 mg/L); S. venezuelae ( 100 mg/L); Streptomyces sp. MBT11 ( 100 mg/L); Control: SFM media (0 mg/L). Concentrations 
A

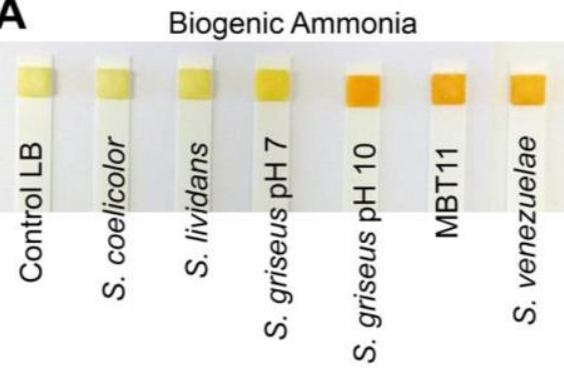

B

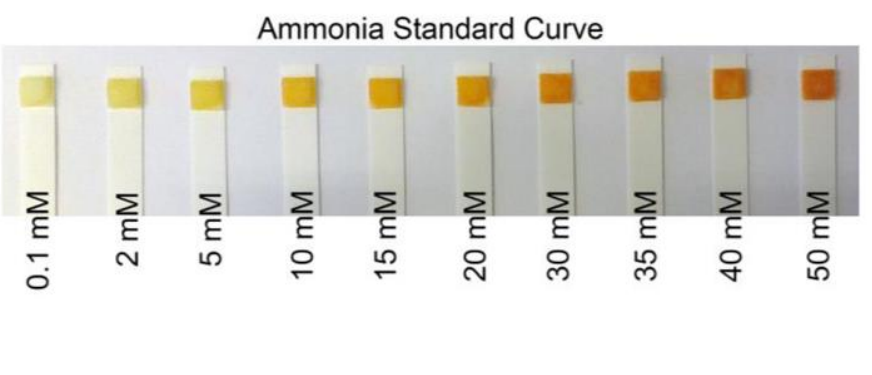

Ammonia Standard Curve

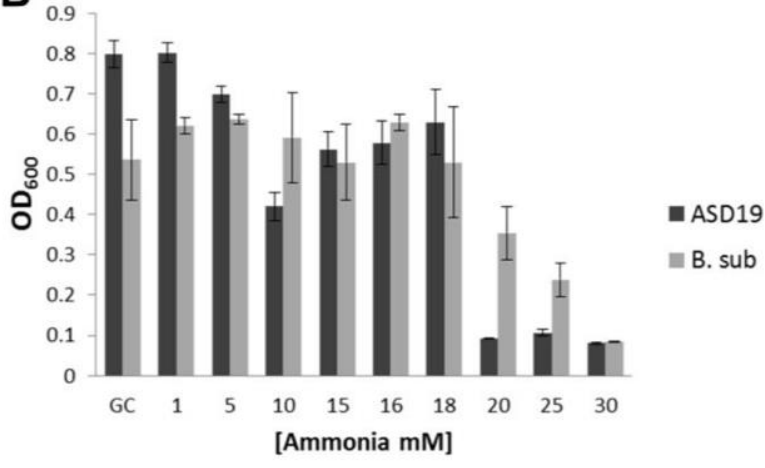

690

691 Figure 3. Bioactivity is caused by ammonia. A. Ammonia quantification from LB agar extracts exposed to Streptomyces VCs (left). Ammonia standard curve from LB agar extract (right). B. Growth of E. coli ASD19 (black) and B. subtilis (gray) under different concentrations of ammonia. 
A

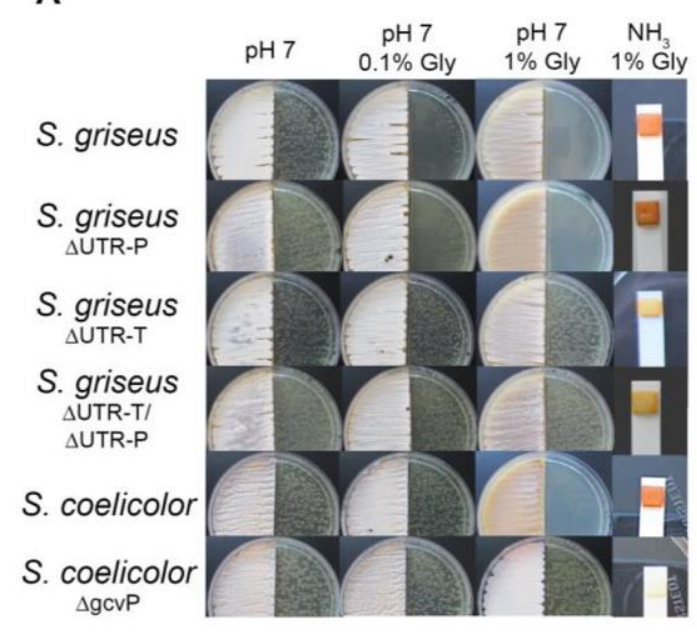

697

698

699

700

701

702

703

704

705

706

707

708

Figure 4. Bioactivity is caused by ammonia in a glycine cleavage-dependent manner. A. Volatile activity and ammonia production by $S$. griseus, $S$. griseus glycine cleavage mutant gcvP (AUTR-P), $S$. griseus glycine cleavage mutant gcvT ( $\Delta \mathrm{UTR}-\mathrm{T})$, S. griseus glycine cleavage double mutant gcvT-gcvP (AUTR-T/AUTR-P), S. coelicolor and the S. coelicolor glycine cleavage system mutant $\Delta g c v \mathrm{P}$. B. Scheme representation of the reactions carried by the glycine cleavage (GCV) system, consisting of: pyridoxal phosphate-containing glycine decarboxylase GcvP (blue); THF-dependent aminomethyltransferase GcvT (yellow); dihydrolipoamide dehydrogenase GcvL; and lipoic acidcontaining carrier protein GcvH. C. Illustration of the induction of volatile antibiosis in different Streptomyces strains when increasing concentrations of glycine are added. 
A E. coli ARM9

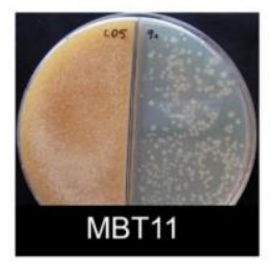

B

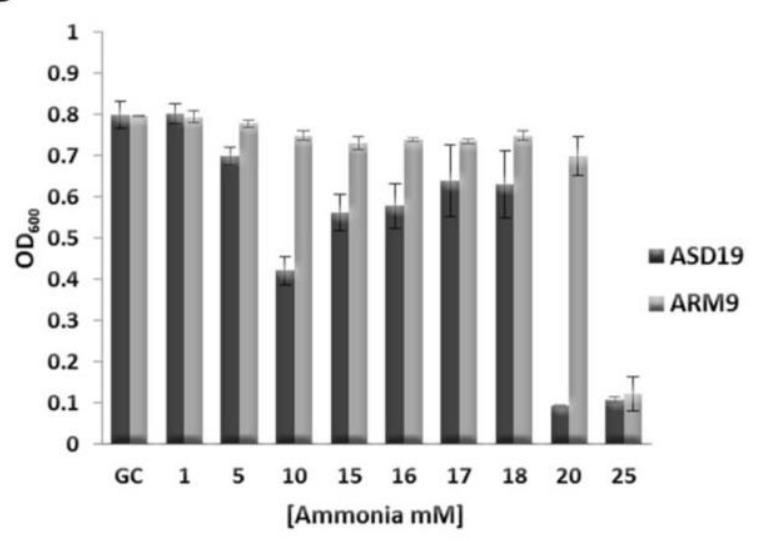

C
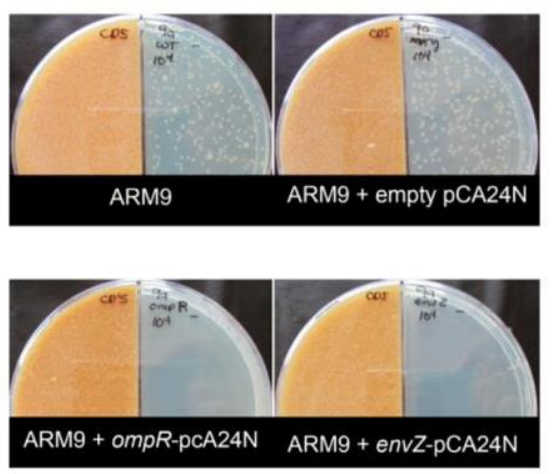

711 Figure 5. Insertion sequences in the E. coli ompR-envZ promoter govern resistance to ammonia. A. (left) spontaneous suppressor mutant ARM9 derived from E. coli strain ASD19 growing under the presence of volatile compounds produced by Streptomyces sp. MBT11; (right) visualization of insertion sequences in-between the 10 and -35 sequences of the ompR/envZ promoter. B. Growth of E. coli strain ASD19 (black) and E. coli strain ASD19 suppressor mutant ARM9 (gray) under the presence of different concentrations of ammonia. C. Growth of suppressor mutant ARM9 and transformants harbouring either empty plasmid $\mathrm{pCA} 24 \mathrm{~N}$, plasmid ompRpCA24N (expressing ompR), or plasmid envZ-pCA24N (expressing envZ). Note that introduction of a plasmid expressing either envZ or ompR makes ARM9 sensitive again to ammonia. 


\begin{tabular}{lcc}
\hline Antibiotic & E. coli & B. subtilis \\
\hline Ampicillin & $\mathrm{NC}$ & + \\
Erythromycin & + & + \\
Kanamycin & + & $\mathrm{NA}$ \\
Tylosin & $\mathrm{NA}$ & + \\
Actinomycin & $\mathrm{NA}$ & + \\
Spectinomycin & + & + \\
Streptomycin & $\mathrm{NA}$ & + \\
\hline
\end{tabular}

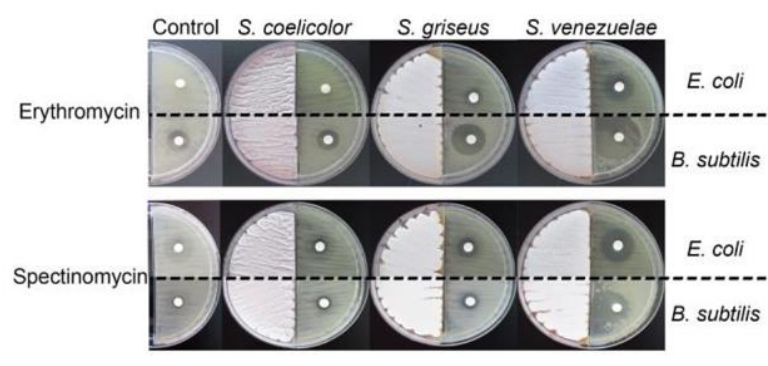

722 Figure 6. Cooperativity between VCs and soluble antibiotics produced by streptomycetes. Left: changes in

723 antibiotic sensitivity caused by the presence of Streptomyces VCs. values indicated increase (+) in halo size. NC,

724 no changes; NA, not active. Right: representative images showing changes in halo size. Streptomycetes were 